Ann. Sci. forest., 1979, 36 (2), 165-173.

\title{
Variabilité phénotypique de l'angle du fil du bois en fonction de la hauteur chez le Pin laricio et le Douglas
}

\author{
Y. BIROT, M. ARBEZ *, P. AZOEUF et R. HOSLIN ** \\ avec la collaboration technique de D. CHARON * et J. BRACH* \\ Station d'Amélioration des Arbres forestiers, I.N.R.A., \\ Ardon, 45160 Olivet \\ * Laboratoire d'Amélioration des Arbres forestiers, I.N.R.A., Domaine de l'Hermitage, \\ Pierroton, 33610 Cestas \\ ** Section d'Application de la Radio-Activité, C.E.A., B.P. 2, \\ Saclay, 91190 Gif sur Yvette
}

\begin{abstract}
Résumé
L'utilisation de radio-isotopes et de l'autoradiographie a permis de mettre en évidence une augmentation de l'angle du fil du bois avec la hauteur pour quinze arbres de deux espèces : Douglas et Pin laricio de Corse. Les déviations sont plus faibles pour le Douglas que pour le Pin laricio.

Faisant l'hypothèse que l'angle du fil du bois serait déterminé par des flux auxiniques, il est suggéré que l'angle du fil du bois du cœur vers l'écorce à $1,30 \mathrm{~m}$ suit la même loi que la variation du haut vers le bas de l'arbre pour un même cerne ; en effet, avec le vieillissement de l'arbre, la distance du pied à la cime (partie où les auxines sont synthétisées) s'accroît également.
\end{abstract}

\section{1. - Introduction}

La fibre torse constitue un défaut grave pour la plupart des utilisations du bois, chez de nombreuses espèces, résineuses ou feuillues. Le caractère héréditaire de ce défaut chez certaines d'entre elles, Pinus radiata (Nicholls, 1964 ; Pederick, 1971), Pinus taeda (Zobel et al., 1968), Pinus pinaster et Pinus nigra ssp. Laricio var. calabrica (Arbez et al., 1978), Larix kaempferi (Mikami et al., 1972), permettrait d'envisager une amélioration par voie génétique.

Cependant, de nombreuses difficultés subsistent car l'angle du fil du bois varie à un niveau donné pour les différents cernes allant du cœur vers l'écorce, mais également de bas en haut du tronc, tout au long d'un même cerne. La plupart des auteurs s'accordent pour reconnaître, au moins chez les conifères, que l'angle du fil du bois évolue avec l'âge de la gauche vers la droite (Northcott, 1957 ; Elliott, 1958 ; Lowery, 1965 ; Noskowiak, 1963 ; Elliott, 1967 ; Nicholls, 1963 ; Krempl, 1965 ; Mikami, 1975) ; Toutefois, à l'intérieur d'une espèce ou d'une population les exceptions sont assez fréquentes (Northcott, 1957 ; Lowery, 1965). Les lois d'évolution de l'angle du fil du bois avec la hauteur semblent moins clairement établies. Plusieurs auteurs (cf. Nicholls, 1963) émettent l'opinion que l'angle du fil du bois augmente avec la hauteur, d'autres 
ont un point de vue inverse (Fielding, 1953; Liese et Ammer, 1962). Elliott (1967) pour le Douglas ef le Tsuga heterophylla note un certain parallélisme entre évolution dans le temps et évolution dans l'espace : l'angle du fil du bois varie de la gauche vers la droite à un niveau donné, il augmente avec la hauteur (de la droite vers la gauche pour un même cerne).

S'inscrivant dans le cadre de l'amélioration génétique de la qualité du bois, l'étude dont les résultats sont présentés a comme objectif de préciser les lois de variation de l'angle du fil du bois avec la hauteur chez deux espèces, le Pin Laricio de Corse (Pinus nigra Arn. ssp. Laricio var. calabrica) et le Douglas (Pseudotsuga menziesii Mirb. Franco).

\section{2. - Matériel et méthode}

\section{1. - Matériel végétal}

Pour le Douglas, l'étude a été menée sur quinze arbres d'une parcelle unitaire d'une plantation comparative de provenances âgée de 25 ans située au Domaine des

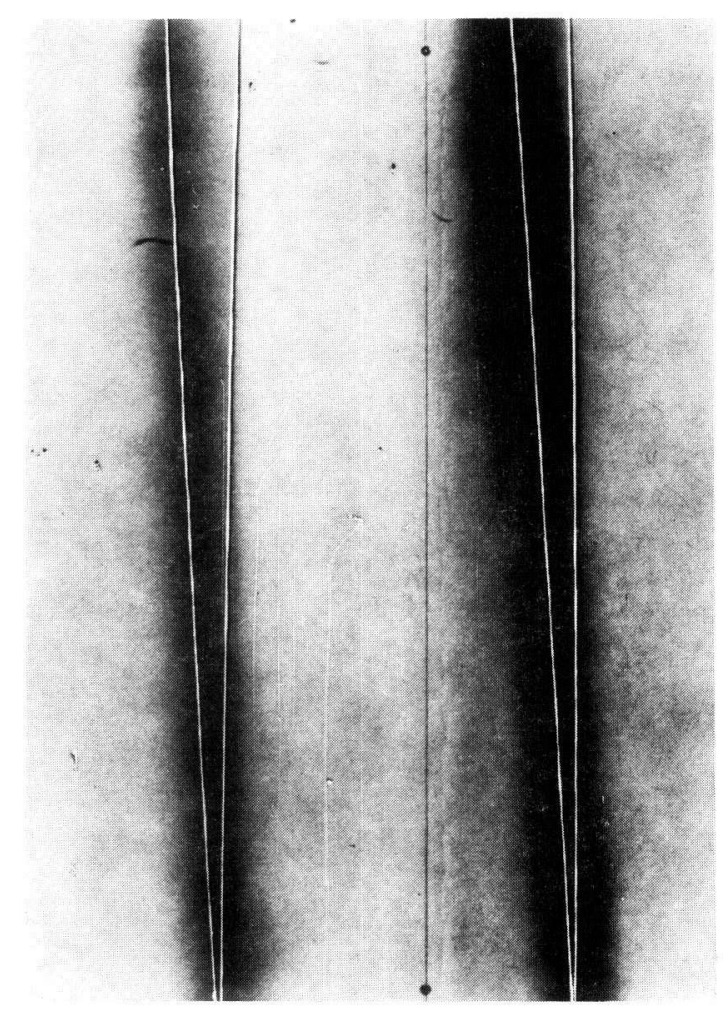

FIG. 1. - Mesure de l'angle du fil du bois : Autoradiographie effectuée sur un Douglas à $9 \mathrm{~m}$ (trace de droite); la trace de gauche correspond à l'injection pratiquée $3 \mathrm{~m}$ plus bas sur la même génératrice.

Fiber angle measurement in a Douglas-Fir tree: Autoradiography following injection of isotope at $9 \mathrm{~m}$ (right hand side mark) ; the light mark on the left arises from an injection $3 \mathrm{~m}$ lower on the same axis line. 
Barres (Loiret) (provenance choisie : ELMA, Washington, U.S.A.) ; les mesures d'angle du fil du bois ont été faites aux hauteurs suivantes : $1,30 \mathrm{~m}, 6 \mathrm{~m}$ et $9 \mathrm{~m}$ pour rester dans la partie du tronc où les branches sont sèches.

Pour le Pin Laricio, l'étude a été menée, toujours aux Barres, sur une plantation âgée de 30 ans environ. Les mesures ont été faites sur quinze arbres aux hauteurs suivantes : $1,30 \mathrm{~m}, 6 \mathrm{~m}$ et $12 \mathrm{~m}$.

\section{2. - Méthode de mesure de l'angle du fil du bois}

La méthode utilisée, non destructive, mettant en œuvre l'autoradiographie de la trace suivie par des radio-isotopes injectés dans la sève ascendante, a été décrite par Keller et al. (1974) et Arbez et al. (1978) la figure I en donne un exemple. Dans le cas particulier le radio-isotope employé est le ${ }^{42} \mathrm{~K}$; l'injection a été pratiquée à chaque niveau de mesure par grimpage sur échelle. Les génératrices ont été repérées au moyen d'un compas forestier (bastringue) muni d'une boussole (parallélisme des branches du compas placé au niveau du point d'injection puis au niveau du point de mesure).

De façon à éviter le grimpage avec la seringue contenant la solution radioactive, on a essayé d'utiliser un isotope à période plus longue, le Rubidium, avec une injection seulement à $1,30 \mathrm{~m}$ : cette tentative a été partiellement infructueuse.

\section{3. - Résultats}

Après changement d'origine (pour surmonter la difficulté de l'orientation différente des angles à gauche ou à droite), on a calculé pour chaque niveau, les moyennes, écart-types, coefficients de variation. On a effectué les comparaisons entre niveaux par la méthode des couples (tests $\nmid$ qui tient comple du fait que les deux données prises sur un même individu ne sont pas indépendantes). Enfin, on a calculé les corrélations intra-arbres entre niveaux.

Les résultats rassemblés au tableau 1 ef schématisés par les figures 2 et 3 peuvent se résumer de la façon suivante :

- Pour les deux espèces (Douglas et Pin Laricio), la valeur moyenne de l'angle du fil du bois reste faible. A $12 \mathrm{~m}$, pour le Pin Laricio, cependant cet angle avoisine les sept grades, ce qui dépasse déjà le maximum généralement tolérable.

- La variabilité intra-arbre (ou contraste d'angle du fil du bois) apparaît forte ; l'amplitude est au maximum de sept grades pour le Douglas et de quatorze grades pour le Pin Laricio. Une telle valeur doit certainement poser des problèmes de transformation du bois. Sur les quinze arbres étudiés, on trouve seulement deux Douglas présentant un contraste supérieur à cinq grades entre 1,30 $\mathrm{m}$ et $9 \mathrm{~m}$; entre $6 \mathrm{~m}$ et $9 \mathrm{~m}$, les contrastes sont au maximum de quatre grades. Pour le Pin Laricio, sur les quinze arbres étudiés, trois présentent un contraste supérieur à cinq grades entre $1,30 \mathrm{~m}$ et $6 \mathrm{~m}$, mais huit arbres présentent un contraste supérieur à sepł grades entre $6 \mathrm{~m}$ et $12 \mathrm{~m}$. 

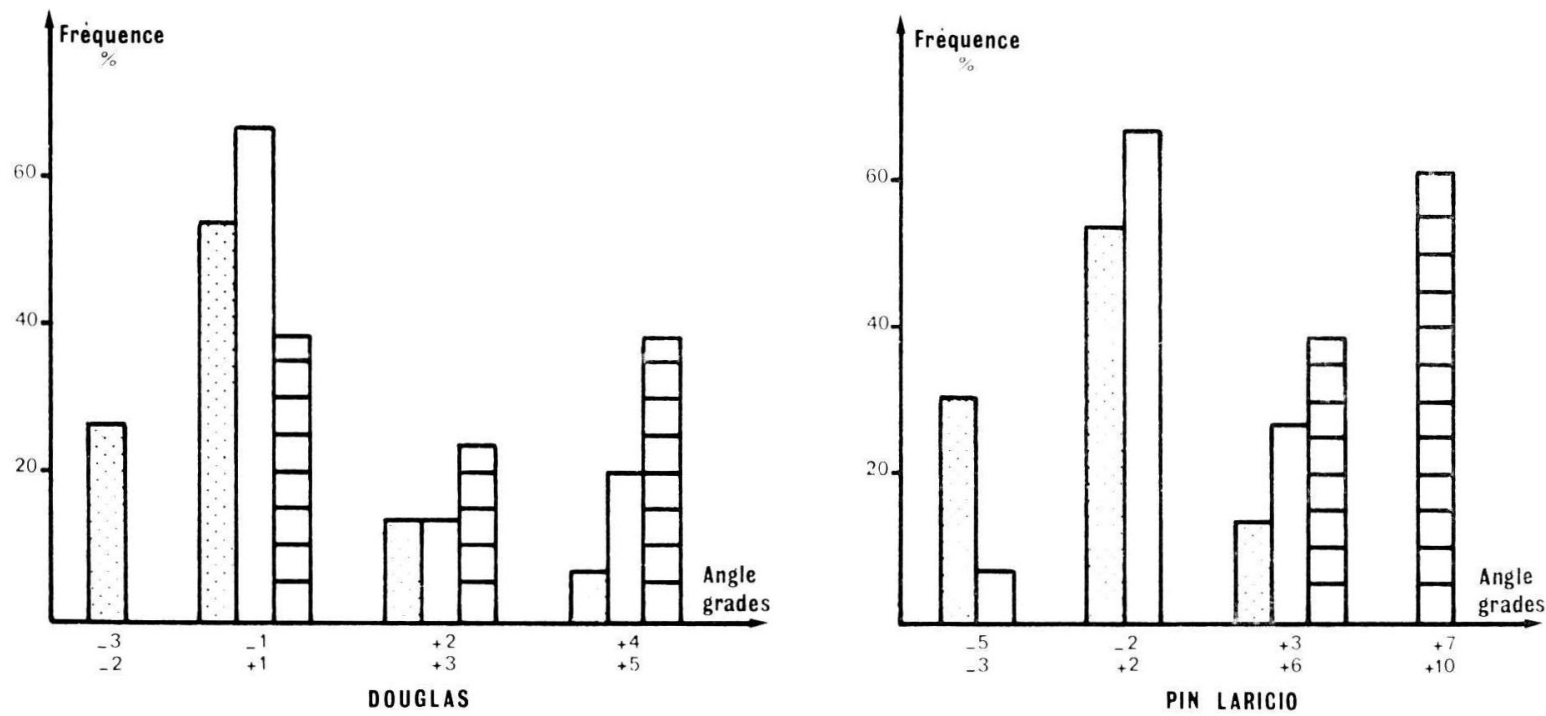

FIG. 2. - Distribution de l'angle du fil du bois aux différentes hauteurs (quinze arbres).

NB : Par convention, les valeurs négatives indiquent une déviation à droite.

Distribution of fiber angle at several levels in the tree (fifteen trees).

NB : Conventionnaly, the algebraic sign means a deviation to the right. 


\section{TABLEAU 1}

Principaux résultats

\begin{tabular}{|c|c|c|c|c|c|c|}
\hline & \multicolumn{3}{|c|}{ Pin Laricio } & \multicolumn{3}{|c|}{ Douglas } \\
\hline & \multicolumn{3}{|c|}{$\begin{array}{l}\text { Hauteur de la mesure } \\
\qquad(\mathrm{m})\end{array}$} & \multicolumn{3}{|c|}{$\begin{array}{l}\text { Hauteur de la mesure } \\
\qquad(\mathrm{m})\end{array}$} \\
\hline & $\begin{array}{c}a \\
1,30\end{array}$ & $\begin{array}{c}b \\
6,00\end{array}$ & ${ }_{12,00}^{c}$ & $\frac{a}{1,30}$ & $\begin{array}{c}b \\
6,00\end{array}$ & $\stackrel{c}{c}$ \\
\hline $\begin{array}{l}\text { Nombre d'arbres mesurés } \ldots \ldots \ldots \ldots \\
\text { Angle moyen }\left({ }^{2}\right) \text { (grades) } \ldots \ldots \ldots \ldots \ldots \\
\text { Ecart-type }\left({ }^{1}\right) \ldots \ldots \ldots \ldots \ldots \ldots \ldots \\
\text { Amplitude entre individus (grades) } \\
\text { Amplitude maximum intra-individus } \\
\text { (grades) } \ldots \ldots \ldots \ldots \ldots \ldots \ldots \ldots \ldots \\
\text { Comparaison de l'angle du fil du bois } \\
\text { aux différentes hauteurs (test } t)\left({ }^{1}\right) \ldots\end{array}$ & $\begin{array}{c}13 \\
-0,61 \\
2,78 \\
8\end{array}$ & $\begin{array}{l}15 \\
1,00 \\
1,96 \\
7\end{array}$ & $\begin{array}{l}13 \\
6,84 \\
2,64 \\
7\end{array}$ & $\begin{array}{c}15 \\
-0,13 \\
1,92 \\
7\end{array}$ & $\begin{array}{l}15 \\
1,53 \\
1,92 \\
5 \\
7\end{array}$ & $\begin{array}{c}13 \\
2,23 \\
1,79 \\
5\end{array}$ \\
\hline
\end{tabular}

1 Calculs réalisés après translation de l'origine à 3 grades à droite (Douglas) et 5 grades à droite Pin Laricio 2 Le signe - indique un enroulement à droite, *, **, *** significatifs aux seuils 0,$05 ; 0,01 ; 0,001$.

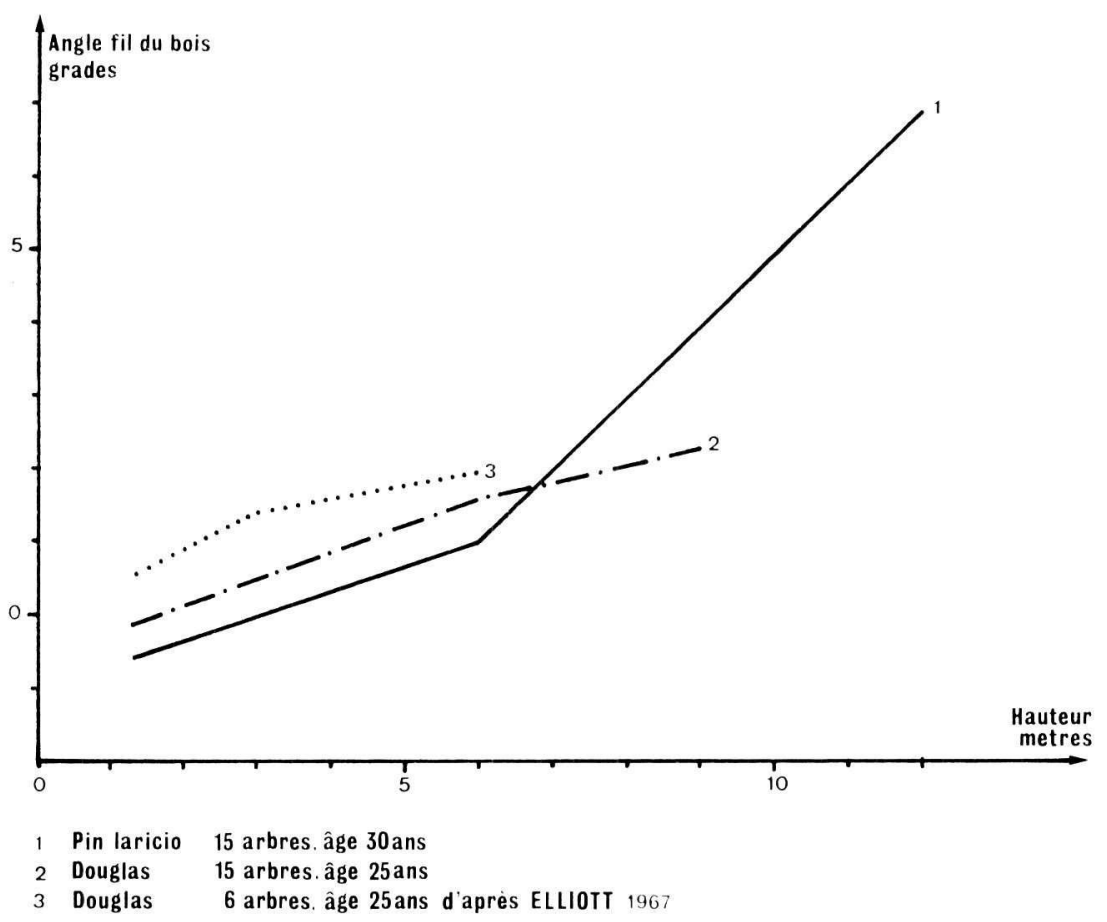

FIG. 3. - Variation de l'angle du fil du bois avec la hauteur. Variation of fiber angle with height. 
Par ailleurs, le nombre d'arbres présentant sur l'ensemble de la bille étudiée un contraste inférieur à cinq grades est de quatre sur quatorze chez le Pin Laricio et de douze sur quatorze chez le Douglas ; il est vrai que le niveau de mesure le plus élevé chez le Douglas n'est que de $9 \mathrm{~m}$ contre $12 \mathrm{~m}$ pour le Pin Laricio.

- La variabilité inter-arbres à un même niveau est assez constante avec la hauteur chez les deux espèces (stabilité de l'écart-type), avec cependant des valeurs souvent plus fortes chez le Pin Laricio.

- L'angle du fil du bois augmente de façon significative avec la hauteur : les déviations en moyenne à droite à $1,30 \mathrm{~m}$, évoluent ensuite vers la gauche lorsqu'on s'élève dans l'arbre. Chez le Pin Laricio on observe une variation brutale entre 6 et $12 \mathrm{~m}$.

- Enfin, les corrélations intra-arbres d'angle du fil du bois ne sont pas significatives (sauf pour le Douglas entre 6 et $9 \mathrm{~m}$ parce que ces deux niveaux sont proches) ; on ne peut prédire à partir de la mesure à 1,30 m la valeur de l'angle du fil du bois à des niveaux supérieurs.

\section{4. - Discussion}

Le défaut de fibre torse apparaît limité chez le Douglas et le Pin Laricio à l'âge étudié, au moins pour la bille de pied. Cela ne veut pas dire, compte tenu de la loi générale d'évolution dans le temps, que la fibre torse n'est jamais un défaut grave chez ces deux espèces. On sait d'autre part qu'il y a des exceptions fréquentes à cette loi, la variabilité entre individus étant très grande.

a) Pour le Douglas, Lowery (1965) montre sur sept cent soixante-quinze arbres d'âge non connu venant des Ełats de Washington, Oregon, Idaho, Montana qu'un quart des échantillons a un angle supérieur à 4.60, l'angle du fil du bois de la majorité des arbres est orienté à droite ; en outre, il n'y a pas d'influence géographique sur l'angle du fil du bois. Northcott (1957) sur cent quarante arbres âgés de première génération en Colombie Britannique montre que 64 p. 100 des individus présentent un angle à droite (évolution de la gauche vers la droite), 19 p. 100, un angle à gauche (accroissement de l'angle de la gauche vers la gauche), 17 p. 100 des arbres présentent d'autres combinaisons ; en outre, cet auteur enregistre des déviations maximums de $16^{\circ}$ vers la gauche et $19^{\circ}$ vers la droite, ce qui est très élevé. Birot (données non publiées) montre sur cent quinze arbres d'un peuplement artificiel français de Douglas âgé de 35 ans (Verreries de Moussans, Hérault) que l'angle du fil du bois à $1,30 \mathrm{~m}$ (cernes extérieurs) est en moyenne de 0,20 grade à gauche, l'écart-type étant de 2,5 : les déviations sont donc faibles.

L'âge auquel l'angle du bois s'annule semble très variable, Elliott (1958) indique 40 ans pour un peuplement de Douglas de deuxième génération, tandis que Northcott (1957) indique un âge compris entre 100 et 300 ans pour des peuplements de première génération.

b) Pour le Pin Laricio de Calabre, les seules données disponibles (Arbez et al. 1978) pour vingt-six arbres âgés de 50 à 150 ans montrent à cet âge que des déviations à $1,30 \mathrm{~m}$ sont généralement faibles à gauche et à droite (deux à cinq grades, moyenne 0,7 grade à droite). Un arbre avait cependant un angle de seize grades à droite ce qui 
est très élevé. A cette exception près, il semblerait que les déviations maximums se soient produites beaucoup plus précocement et à gauche, probablement entre 15 et 30 ans.

La variabilité de l'angle du fil du bois entre arbre et intra-arbre semble importante ce qui est souligné par de nombreux auteurs : Elliott (1967) et surtout Mikami et Nagasaka (1975) qui en donnent de bons exemples pour le Mélèze du Japon (Larix kempferi). Ceci limiterait la portée générale des lois de variation dans le temps et dans l'espace et diminueraî l'efficacité de la sélection de population ou d'individus.

L'augmentation de l'angle du fil du bois avec la hauteur apparaît de façon claire, confirmant les résultats acquis par Elliott (1967) sur six individus de deux espèces : Douglas et Tsuga heterophylla. En moyenne ces variations sont quantitativement assez modérées si l'on se limite à la bille du pied, ceci étant vrai surtout pour le Douglas. Ces résultats expliqueraient pourquoi dans leur étude antérieure Arbez et al. (1978) sur Pin Laricio de Calabre, l'angle du fil du bois à 1,30 m chez les ramets (greffes) était toujours à gauche (supérieur à celui des ortets et en valeur absolue supérieur à celui de leurs descendants issus de graines) ; les greffes ont en effet été prélevées dans la cime des ortets. Ces résultats confirment également les hypothèses faites par Nicholls et al. (1976) pour Pinus radiata. Ces auteurs ont établi que l'angle du fil du bois mesuré sur des ortets âgés à 1,30 m (niveau A) était significałivement plus faible que lorsqu'il était mesuré sur les mêmes ortets dans la partie supérieure du tronc (niveau B). L'angle du fil du bois mesuré sur des plants bouturés (ramets : C) issus de la cime de l'ortet avait des valeurs comparables à celui mesuré dans le haut des ortets (B). En outre, la diminution avec l'âge de la gauche vers la droite de l'angle du fil du bois était plus forte au niveau A qu'au niveau $B$ ou que pour le matériel $C$. Les différences ortets-ramets n'étaient plus significatives lorsque les ortets étaient jeunes. II s'agirait là de phénomènes résultant d'effets de topophysis $(*)$.

Cette augmentation de l'angle du fil du bois avec la hauteur serait expliquée par l'hypothèse de Maddern-Harris (1973) admettant le rôle des auxines pour orienter les fibres ; la déviation par rapport à l'axe du tronc serait plus forte au voisinage de leur lieu de synthèse : la cime.

Finalement, cette hypothèse conforterait celle émise par Elliott (1967) qui admettait un certain parallélisme entre variation de l'angle du fil du bois dans le temps et dans l'espace. L'existence de deux phénomènes concomittants lors de la croissance de l'arbre pourrait expliquer l'évolution avec l'âge enregistrée à 1,30 m : augmentation à gauche suivie d'une diminution progressive, en la rapprochant de l'accroissement de la masse foliaire qui synthétise l'auxine puis de son éloignement du pied de l'arbre. Néanmoins, ce schéma ne rend pas compte du passage à droite observé chez la plupart des individus âgés. La même hypothèse vaut pour les fibres d'un même cerne lorsqu'on se déplace du haut vers le bas de l'arbre. Tout ceci mériterait d'être vérifié et approfondi par des expériences spécialisées (élagage des branches vertes, ébourgeonnement).

Ceci serait également en accord avec les résultats de Krempl (1965) qui montre sur l'Epicéa que l'angle du fil du bois d'une branche est supérieur à celui du tronc au niveau d'insertion de cette branche.

* Topophysis : Persistance de caractères non génétiques pendant un certain nombre d'années chez les plants issus de multiplication végétative en relation avec la nature (position dans l'ortet, âge) des boutures ou greffons utilisés.

Annales des Sciences forestières. - 1979 
Enfin, il faut noter l'absence de corrélation significative entre l'angle du fil du bois mesuré à différents niveaux dans un arbre ; la détermination faite à un niveau donné n'a donc pas de valeur prédictrice pour l'ensemble de la bille, ceci étant surtout net chez le Pin Laricio. On peut remarquer d'abord que l'importance économique de la bille de pied diminue la portée de l'absence de la corrélation entre niveaux. En outre, ce fait traduit que si la loi générale est bien une augmentation de l'angle du fil du bois avec la hauteur, le contraste de densité varie suivant les individus, d'où la possibilité de sélectionner des individus à faible contraste de bas en haut du tronc. L'objectif est finalement de sélectionner des individus dont l'angle du fil du bois à $1,30 \mathrm{~m}$ est faible et dont le contraste est faible. Ceci semble possible, en particulier sur le Douglas. Une étude antérieure réalisée sur un Douglas âgé d'environ 80 ans en forêt communale d'Epinal montre qu'entre $1,30 \mathrm{~m}$ et $24 \mathrm{~m}$ de hauteur (les niveaux intermédiaires mesurés étant $6 \mathrm{~m}$ et $12 \mathrm{~m}$ ), le contraste n'excède pas deux grades, l'angle du fil du bois à 1,30 m étant nul. La hauteur de l'arbre dépassait $45 \mathrm{~m}$.

\section{5. - Conclusion}

Les résultats exposés mettent en évidence l'augmentation de l'angle du fil du bois avec la hauteur. Cependant, et comme dans le cas de l'évolution de ce caractère avec l'âge, il s'agit d'une loi générale, les variabilités inter et intra-arbre semblent importantes. On peut également souligner l'intérêt du Douglas du fait de ces faibles valeurs moyennes d'angle du fil du bois : c'est encore une qualité supplémentaire à porter au crédit de cette essence.

Dans la mesure où la méthodologie de détermination de l'angle du fil du bois sur carottes progresserait, on pourrait envisager de sélectionner des individus à faible contraste d'angle de fil du bois à un même niveau et entre niveaux. Si l'on utilise la voie végétative, les effets de topophysis, mis en évidence par Nicholls et al. (1977) sur Pinus radiata pourraient cependant compliquer le problème. Si l'on utilise la voie générative, il faudrait vérifier l'hérédité de ces critères.

Dans la mesure où l'on pourrait démontrer l'existence de parallélisme dans l'évolution de l'angle du fil du bois du cœur vers l'écorce et du sommet vers le bas, il y aurait possibilité de sélection car nous savons mesurer l'angle du fil du bois sur les cernes extérieurs de bas en haut de l'arbre, comme cette étude le montre ; ceci éviterait la mesure difficile de l'angle du fil du bois à différents âges.

On peut cependant s'attendre à ce qu'une sélection précoce soit difficile à mettre en œuvre, sauf à éliminer les individus présentant un angle des fibres ou un contraste vraiment excessif. Compte-tenu du coût de ces opérations, même limitées à la bille de pied, cette procédure devrait concerner l'étape ultime de la sélection.

Des recherches de type explicatif semblent encore nécessaires pour déterminer les mécanismes contrôlant l'angle du fil du bois et sa variation dans le temps et dans l'espace. 


\section{Summary}

\section{Phenotypic variation of spiral grain along the stem in Corsican Pine and Douglas-Fir}

By using radio-isotope injection and autoradiography a pattern of spiral grain variation with height has been found in fifteen trees of both species i.e. fiber angle increasing from the bottom to the top. Deviation was less pronounced in Douglas-Fir than in Corsican Pine.

Assuming that fiber angle is controlled by auxin flow it is suggested that variation of fiber angle from the center of the trunk to the bark at breast height follows the same pattern as the variation from the top to the bottom of the tree, because with increasing age, the distance to the crown, where auxins presumably originate, also increases.

\section{Références bibliographiques}

ARBEZ M., BARADAT Ph., BIROT Y., AZOEUF P., HOSLIN R., (1978). Hérédité de l'angle du fil du bois mesuré à l'aide d'un traceur radio-actif chez le Pin maritime et le Pin Laricio. Journal Canadien de la Recherche Forestière (sous presse).

ELLIOTT G. K., (1957). Spiral grain in second growth Douglas Fir and Western Hemlock., M. F. Thesis UBC p. 66.

ELLIOTT G. K., (1958). Spiral grain in second growth Douglas-Fir and Western Hemlock. Journ. For. Prod. Res. Soc., VIII (7), 205-211.

ELLIOTT G. K., (1967). Some problems of spiral grain with special reference to conifers, IUFRO Kongress Munich 1967, pp. 413-435.

FIELDING J. M., (1953). Variations in Monterey Pine. For. and timber Bur. Aust. Bull., 31.

KELLER R., AZOEUF P., HOSLIN R., (1974). Détermination de l'angle de la fibre torse d'arbres sur pied à l'aide d'un traceur radioactif. Annales des Sciences forestières, 31, pp. 161-169.

KENNEDY R. W., WARREN N. G., (1969). Within tree variation in physical and chemical properties of Douglas-Fir. Deuxième consultation mondiale sur l'amélioration des Arbres forestiers FAO/IUFRO Washington.

KREMPL H., (1965). Spiral growth in the log and branchwood of spruce. Presented at Meeting of IUFRO Sect. 41, Melbourne.

LIESE W., AMMER V., (1962). Anatomical studies on extreme twisted pine wood., Holz als Roh und Werkstoff 20, 339-346.

LOWERY D. P., (1965). A spiral grain classification system and its application. Forest. Products. Research Society Meeting New-York.

LOWERY D. P. (1965). Spiral grain patterns in Douglas-Fir., Proceedings, Montana, Academy of Sciences, Missoula 25, 62-67.

MIKAMI S., NAGASAKA K., (1975). Selection for minimizing spiral grain in Larix leptolepis. Bulletin of the Government Forest Experiment Station Tokyo, n ${ }^{\circ} 276$, pp. 3-22.

MIKAMI S., WATANABE M., OHTA M., (1972). Titre inconnu. Journal of Japanese Forestry Society, 54 p., 213-217.

NICHOLLS J. W. P. (1963). The relation of spiral grain to wood quality. In Proc. Sect. 41, IUFRO Madison U.S.A.

NICHOLLS J. W. P., PAWSEY C. K., BROWN A. G., (1976). Further studies on the ortet-ramet relationship in wood characteristics of Pinus radiata., Silvae Genetica 25, Heft 2, Pp. 73-79.

NORTHCOTT P. L., (1957). Is spiral grain the normal growth pattern. For. Chron. 33 (4), 335-352. 